\title{
Formal Syntax and Semantics of Case Stacking Languages
}

\author{
Christian Ebert \\ Lchrstuhl für Computerlinguistik \\ Universität Heidelberg \\ Karlstr. 2 \\ D-69117 Heidelberg
}

\author{
Marcus Kracht \\ II. Mathematisches Institut \\ Freie Universität Berlin \\ Arnimallee 3 \\ D-14195 Berlin
}

\begin{abstract}
In this paper the phenomenom of case stacking is investigated from a formal point of view. We will define a formal language with idcalized case marking behaviour and prove that stacked cases have the ability to encode structural information on the word thereby allowing for unrestricted word order. Furthermore, the case stacks help to compute this structure with a low complexity bound. As a second part we propose a compositional semantics for languages with stacked cases and show how this proposal may work for our formal language as well as for an example from Warlpiri.
\end{abstract}

\section{Introduction}

Case stacking is a phenomenom that occurs in many Australian languages (such as Wartpiri and Kayardild) and e.g. Old Georgian. Case stacking is known to pose problems for the treatment of case in many formal frameworks today $^{1}$. In (Nordlinger, 1997) the problem was attacked by extending the framework of LFG. Nordlinger claims that case morphology can construct grammatical relations and larger synlactic contexts.

In Section 2 we will introduce an ideal languge, which exhibits perfect marking. This language captures Nordlinger's idea of case as constructors of grammatical relations, but is independent of any syntactic framework. Wo will prove of this language that the case stacks provide all the information needed for reconstructing the functor-argument relations and the syntactic context a word appears in. Additionally, since structure lies encoded in these caso stacks there is no need to assume any phrase structure

\footnotetext{
'see (Nordlinger, 1997) and (Malonf, 1999) for a discussion on $\mathrm{LFG}$ and $\mathrm{HI}^{\prime} \mathrm{SG}$ respectively.
}

or restriction on word order. At the end of this section we consider the computational complexity of our language and draw some conclusions about grammar formalisms that are able to gencrate it.

In Section 3 we propose a compositional semantics for our case stacking languages. Unlike Montague semantics, where the management of variables is not made explicit, we will use referent systems to keep track of variables and to make semantic composition efficently computable ${ }^{2}$. The proposal will be applied to our formal language and to an example from Warlpiri.

\section{Syntax}

In this section a perfectly case marked formal language will be defined and investigated. The definition of this language is based on terms consisting of functors and arguments and thus cases will be taken to mark arguments.

In the following we let $\mathbb{N}$ denote the set of non-negative integers and the concatenation of strings, which is often omitted. We shall use typewriter font to denole true characters in print for a formal language.

\subsection{Basic Definitions}

An abstract definition of terms runs as follows. Let $F^{\prime}$ be a set of symbols and $\Omega: F \rightarrow \mathbb{N}$ a function. The pair $\langle F, \Omega\rangle$ is called a signature. We shall often write $\Omega$ in place of $\langle F, \Omega\rangle$. An element $f \in F$ is called a functor and $\Omega(f)$ the arity of $f$. We let $\omega:=\max \{\Omega(f) \mid f \in F\}$ denote the maximal arity. Terms are denoted here by strings in Polish Notation, for simplicity.

Definition 1. Let $\Omega$ be a signalure. A term over $\Omega$ is inductively defined as follows.

\footnotetext{
${ }^{2}$ see (Vermeulen, 1995) and (Kracht, 1999) on these issues.
} 
1. If $\Omega(f)=0$, then $f$ is a term.

9. If $\Omega(f)>0$ and $t_{i}, 1 \leq i \leq \Omega(f)$, are lerms, so is $f t_{1} \ldots t_{\Omega(s)}$.

'The set $C$ of' case markers will be the set $\{1, \ldots, \omega\}$, which we assmuse to be disjoint, from $F$. Given a term, each finctor will be case marked acecording to the argunent position it occupies. 'This is achieved thronght the notion of a unit, which consists of a finctor and a sequence of case markers callod case stuch

Definition 2. Let t be a term ouer a signature S. The corresponding bag, $\Delta(l)$, is industively defined as follows.

1. If $t-f$, then $\Delta(t):=\{t\}$.

Q. If $t=f t_{1} \ldots t_{n,}$, then $\Delta(t)::=\{f\} U$ $\bigcup_{i=1}^{n}\left\{z^{\prime} i \mid z \in \Delta\left(t_{i}\right)\right\}$.

An clement f $\gamma \in \Delta(t)$ is called a unit and $\gamma \in$. $C^{*}$ its case stack.

For example, if $f, g$, and $x$ are functors of arity 2, 1 and (), resjectively, the hag, $\Delta(f a g x)$ is $\{f, x 1, g 2, x 12\}$. The moaning of a unit $x 12$ conld be described by ' $x$ is the finctors of the first argument af the second argmunent of the tormon".

Definition 3. Let t be a term over a stghature S?, $\Delta(t)$ the comresponding bay and $\Delta(t)=:\left\{\delta_{i} \mid\right.$ $i \leq n\}$ an arbitrary enumeration of its units. Then the string $\delta_{1}-\delta_{2}-\ldots \sim \delta_{n}-\delta_{n}$ is suid to be a $\Delta(t)$-string.

Sone of the $\Delta(f x g x)$-strings are e.g. $\mathrm{f} \times 1 \mathrm{~g} 2 \times 12$ and $\mathrm{g} 2 \times 1 \mathrm{f} \times 12$. Wo are now prepared to define a formal language over the alphabet $r \cup C$ by collecting all $\Delta(t)$-strings for a given signature:

Definition 4. Let $\Omega$ be a signature. The ideal case marking language $\mathcal{I C M} \mathcal{L}^{\mathrm{S}}$ over this signature consists of all $\Delta(t)$-strings such that $t$ is a term over $\Omega$.

\section{2 'Trees and Unique Readability}

There is a strong correspondence between bags and labelled trees since ase stacks cam be identified with tree aldresses:

Definition 5. A noncmpty finite set 1$) \leq \mathbb{N}_{+}^{*}$ is a tree domain if the following hold:
1. $\epsilon \subset D$.

8. If $d_{1} d_{2} \in D$ then $d_{1} \in D$ ).

3. If di $\in D, i \in \mathbb{N}$ then di $\in D$ for all $j<i$.

The clcments of a bes domain are called tree addresses. A $\Omega$-labclled tree is a pair $\langle(D), \tau\rangle$ such that $D$ is a tree domain and $\tau: D \rightarrow H^{\circ}$ a labelling function such that the number of dewughters of $d \in D$ is exactly $\Omega(\tau(d))$.

To formalize the correspondence we define a function 7 that assigns every bag $\Delta(t)$ a $\Omega$ labelled tree:

$$
\begin{array}{r}
T\left(\left\{\mathrm{f}_{1} \gamma_{1}, \ldots, \mathrm{f}_{n} \gamma_{n}\right\}\right):-\left\langle\left\{\gamma_{i}^{R} \mid 1 \leq i \leq n\right\},\right. \\
\left.\tau: \gamma_{i}^{h} \mapsto f_{i}(1 \leq i \leq n)\right\rangle
\end{array}
$$

'l'Ie: function 'T' reverses the case stacks of all units to get a set of tree acklresses. Then the? finctor of the nuit, is assigned to the tree antdress. Fig. if the bag contains a tuil g321 the resulting tree domain will contain a taee address 123 and the labelling function will assign $g$ to it.

Similarly one can define an inverse finnetion assigning a bag to oach s-labelled tree. Thens there is a bijection between $\Omega$-labelled trees and bags. 'Therefore different bags correspond to different ordered labelled trees. 'This shows that we have mirge rearability for bags and since

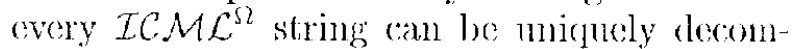
prosed into its mits wo may statio the following proposition.

Proposition 6. Let $\Omega$ be a signature. Then ev-

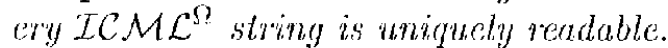

\subsection{Pumpability and Semilinearity}

Wo will first consider the property of being finitely pumpable as defined in (Groenink, 1997).

Definition 7. A language $I$ is finitely pumpable if there is a constant c such that for any $w \in L$ with $|w|>c$, there are a finite number $k$ and strings $u_{0}, \ldots, u_{k}$ and $v_{1}, \ldots, v_{k}$ such that $w=u_{0} v_{1} u_{1} v_{2} u_{2} \cdots v_{k} u_{k}$ and for each $i, 1 \leq\left|v_{i}\right|<$ c and for any $p \geq 0$ the string $u_{0} v_{1}^{p} u_{1} v_{2}^{p} u_{2} \cdots v_{k}^{p} u_{k}$ belongs to $\mathrm{L}$.

Proposition 8. Fet $\Omega$ be a signalare. Then ICML $\mathcal{L}^{S 2}$ is not jinitely pumpable. 
Proof. It is easy to observe that the pumpable parts cannot contain a functor since that would lead to pumped strings containing the same units more than once. Hence the number of units camnot be increased by pumping and all pumpable parts must consist of case markers solely. But since the length of an $\mathcal{I C M} \mathcal{L}^{\mathrm{S}}$ string consisting of a fixed number of units is bounded each pumpable string could be purnped up such that it exceeds this bound. Thus $\mathcal{I C M \mathcal { L } ^ { \Omega }}$ is not finitely pumpable at all.

Now we are concerned with semilinearity.

Definition 9. Let $M \subseteq \mathbb{N}^{n}$. Then $M$ is a

1. linear set, if for some $k \in \mathbb{N}$ there are $u_{0}, \ldots, u_{k} \in \mathbb{N}^{n}$, such that $M=\left\{u_{0}+\right.$ $\left.\sum_{i=1}^{k} n_{i} u_{i} \mid n_{i} \in \mathbb{N}\right\}$,

2. semilinear set, if for some $k \in \mathbb{N}$ there are lincar sets $M_{1}, \ldots, M_{k} \subseteq \mathbb{N}^{n}$, such that $M=\bigcup_{i=-1}^{k} M_{i}$.

A language $I$, over an alphabet $\Sigma=\left\{w_{i} \mid\right.$ $0 \leq i<n\}$ is called a semilinear language if its image under the Parikh mapping is a semilinear sel, where the Parikh mapping $\Psi$ : $\Sigma^{*} \rightarrow \mathbb{N}^{n}$ is defined as follows:

$$
\begin{array}{llll}
\epsilon & \mapsto\langle 0, \ldots, 0\rangle & & \\
w_{i} & \mapsto e^{(i)} & \text { for } 0 \leq i<n \\
\alpha \beta & \mapsto \Psi(\alpha)+\Psi(\beta) & \text { for all } \alpha, \beta \in \Sigma^{*}
\end{array}
$$

where $e^{(i)}$ is the $i+1$-th unit vector, which consists of zeros except for the $i$-th component, which is 1 .

Note that - given a term $t$ - the Parikl imago of all $\Delta(t)$-strings is the same since these are just concatenations of different permutations of the units in $\Delta(t)$.

In the following we make use of a proof technique used in (Michaelis and Kracht, 1997) to show that Old Georgian is not a scmilinear language. We cite a special instance of a proposition given therein:

Proposition 10. Let $P(k)=\frac{\omega}{2} k^{2}+\frac{2-\omega}{2} k$ and $M$ be a subset of $\mathbb{N}^{n}$, where $n \geq 2$, which has the properties

1. For any $k \in \mathbb{N}_{+}$there are some numbers $l_{2}^{(k)}, \ldots, l_{n-1}^{(k)} \in \mathbb{N}$ for which the n-tuple. $\left\langle k, P(k), l_{2}^{(k)}, \ldots, l_{n-1}^{(k)}\right\rangle$ belongs to $M$.
2. For any $k \in \mathbb{N}_{+}$the value $P\left(k_{-}\right)$provides an upper bound for the second component $l_{1}$ of any $n$-tuple $\left\langle k, l_{1}, \ldots, l_{n-1}\right\rangle \in M$ (that means $l_{1} \leq P(k)$ for any such n-tuple).

Then $M$ is not semilinear.

In order to investigate the semilinearity of $\mathcal{I C M C}^{\Omega}$ we choose distinct symbols $f, x \in F$, such that $\Omega(f)=\omega$ and $\Omega(x)=0$. We shall construct terms $s_{i}$ by the following inductive definition:

1. $s_{0}:=x$

2. $s_{n}:=f\left(s_{n-1}, x, \ldots, x\right) \quad$ for $n>0$

It is casy to observe that by virtue of construction $s_{n}$ consists of $n$ leading functors $f$ and that in each iteration tho number of $x$ increases by $(\omega-1)$.

Lemma 11. Let $F \cup C=\{f, 1, x, 2, \ldots, \omega$, $\left.f_{1}, \ldots, f_{|F|-2}\right\}$ be an enumcration of the alphabet underlying $\mathcal{I C M C}^{\Omega 2}$, where $f_{1}, \ldots, f_{\mid F_{\mid}-2}$ are the remaining functors in $F-\{f, x\}$ Then the Parikh image of some $\Delta\left(s_{n}\right)$-string $\delta_{n}$ is

$$
\begin{aligned}
\Psi\left(\delta_{n}\right)=\langle n, & \frac{\omega}{2} n^{2}+\frac{2-\omega}{2} n, \\
& (\omega-1) n+1, \underbrace{n, \ldots, n}_{\omega-1}, \underbrace{0, \ldots, 0}_{|F|-2}\rangle
\end{aligned}
$$

Furthermore, $\frac{\omega}{2} n^{2}+\frac{2-\omega}{2} n$ imposes an upper bound on the second component of $\Psi\left(\delta_{n}\right)$.

Proof. The first part of the lemma can bo proved in a straightforward way by induction on $n$. 'The claim on the upper bound follows from the observation that the number of occurrences of case marker 1 can be maximized by repcated embedding of terms in the first argument position.

Proposition 12. Let $\Omega$ be a signature. Then $\mathcal{I C M} \mathcal{L}^{\Omega}$ is not semilinear.

Proof. Let $n=\omega+|F|$ and consider the linear, and henco semilinear, set $R:=$

$$
\left\{\left(n_{2}(\omega-1)+1\right) e^{(2)}+\sum_{i=0, i \neq 2}^{\omega+2} n_{i} e^{(i)} \mid n_{i} \in \mathbb{N}\right\}
$$


Then the full preimage $L_{R}$ of $R$ under the Parikh maj) consists of all staings which contain $n_{2}((\omega-1)+1)$ occurrences of the symbol $\mathrm{x}$ (where $n_{2}$ is any number) and any number of occurrences of the symbols $f, 1, \ldots, \omega$, and no other symbols. We define the language $I_{M}$ as the set of all strings belonging to $L_{R}$ and the ideal case marking languages. 'J'hen $L_{M}$ contains all $\Delta\left(s_{n}\right)$-strings.

Considering the Parikh inage $M$ of $L_{M}$ we get:

$$
\begin{aligned}
& M=\Psi\left[L_{M}\right]=\Psi\left[I_{R}\right] \cap \Psi\left[\mathcal{I C M} \mathcal{L}^{\Omega}\right] \\
& =R \cap \Psi\left[\mathcal{I C} \mathcal{M L}^{\Omega}\right]
\end{aligned}
$$

because of the definition of $L_{R}$ as the full preinage of $R$. But then the set $M$ fulfills the conditions of Proposition 10 due to Iemma 11. Hence $M$ is not semilinear. Since $R$ is semilinear by definition and semilinearity is closed under intersection $\mathcal{I C M} \mathcal{L}^{\Omega}$ is not semilinear.

\subsection{Computational Complexity}

In this subsection the computational complexity of $\mathcal{I C M \mathcal { L } ^ { S 2 }}$ is considered. The resultis are achieved by defining a 3-tape-Turing machine acceptor (depending on a given signature) that recognizes $\mathcal{I C} \mathcal{M L}^{\Omega}$.

Proposition 13. Let $\Omega$ be a signature. Then

$$
\begin{aligned}
& \mathcal{I C M L} \mathcal{L}^{\Omega} \in \operatorname{DTTME}(n \sqrt{n} \log n) \\
& I C \mathcal{M L}^{\Omega 2} \in D S I^{\prime} A C H(n) \text {. }
\end{aligned}
$$

Proof. In the following we let $n$ denote the length of the input string. The 'Juring machine algorithm can be subrlivided into three main partis:

1. The input string is segmented into its units: The algorithm steps through the input and adds separation markers in between two units. This can be done in $O(n)$ time.

2. The units are sorted according to their caso stacks: More formally a \%-way straight merge sort is performed. This sorting algorithm is known for its worst case optimal complexity: it performs the sort of $k$ keys in $O(k \log k)$ steps. In our case the keys are units and thus thoir number is clearly bounded by $n$. The additional square root: factor comes from the comparison step. One can show that the maximal length of a case stack occuring in an $\mathcal{I C M} \mathcal{L}^{S l}$ string of length $n$ is bounded above by $O(\sqrt{n})$. Hence a comparison of two units takes at most $O(\sqrt{n})$ steps. Thus the overall complexity of the sorting part is $O(n \sqrt{n} \log n)$.

3. The sorted secpuence of units is checked: The algorithm successively genorates case stacks according to the functors it has read. Each case stack is compared to the wnit of the input. If they coincide the algorithm advances to the next unit on the input and generates the next case stack. After all case stacks have been generated the whole input string must have been worked through. In this case the algorithm arcepts. This can be done in $O(n)$ time.

Summing up the complexitios of these three parts shows that the time complexity is as claimed in the proposition. Furthermore, the algorithm uses only the cells needed by the input plus at most $k_{i}-1$ colls for additional separation markers (due to the first part), where $k$ is the number of units the input string consists of. This shows that the space complexity is lincar.

\subsection{Discussion}

A first conclusion we may draw is that cases have the ability to construct the context they appear in. $\mathcal{I C M} \mathcal{L}^{\Omega}$ strings encode the samo structural information as ordered labelled trees do thereby allowing unconstrained order of units. Arlditionally cach such string can be read unambigously. This was shown by means of a bijection between bags and ordered labelled trees.

The fact that ideal case marking languages are neither finitely pumpable nor semilinear moans that they fall out of a lot of hicrarchies of formal languages. As (Weir, 1988) shows, multicomponent tree adjoining grammars ${ }^{3}$ generate only semilincar languages. Consequently, ideal case marking languages are not MCTALs. Howcver, (Groenink, 1997) defines a class of grammars, called simple literal movement grammars,

\footnotetext{
${ }^{3}$ and hence linear context-free reurite systerns, which are shown to be weakly equivalent to MCIAGs in (Weir, 1988)
} 
which generate all and only the PTIME recognizable languages. Idcal case marking languages should therefore be generated by some simple literal movement grammar.

We note furthermore that the (theoretical) time complexity is significantly better than the best known for recognizing context-free grammars. In fact, we implemented a practically applicable algorithm which constructs the corresponding tree out of a given $\mathcal{I C M \mathcal { L } ^ { \Omega }}$ string in linear time (in average).

\section{Semantics}

We are now going to propose a semantics for languages with stacked cases. The basic principle is rather easy: we are going to identify variables by case stacks thereby making use of referent systems.

\subsection{Referent Systems}

The semantics uses two levels: a DRS-level, which contains DRSs, and a referent level, which talks about the names of the referents used by the DRS. Referent systems were introduced in (Vermeulen, 1995). We keep the idea of a referent system as a devicc which administrates the variables (or referents) under morge. The technical apparatus is however quite different. In particular, the referent systems we use define explicit global string substitutions over the referent names.

There is one additional symbol o. It is a variable over names of referents. If we assume that a functor $g$ has meaning $g$ a simple lexical entry for $g$ looks like this:

\begin{tabular}{c}
$\mid \mathrm{g} /$ \\
\hline $0: 0$ \\
\hline $0 \doteq \mathrm{g}\left(1 \frown 0,2 \frown 0, \ldots, \Omega(g)^{\frown 0} \mathrm{o}\right.$ \\
\hline
\end{tabular}

Here, the upper part is the referent system, and the lower part an ordinary DRS, with a head section, containing a set of referents, and a body section, containing a set of clauses. This means that the semantics of a functor $g$ is given by the application of $g$ to its arguments. However, instcad of variables $x, y$, etc. we find $1 \frown 0,2 \frown 0$, etc. The semantics of a 0 -ary functor $x$ and a case marker, say 2, are:

\begin{tabular}{|c|}
\multicolumn{1}{c}{$\frac{1 / 2 /}{0: 0}$} \\
\hline$\varnothing$ \\
\hline $0 \doteq x$ \\
\hline $0: 2-0$ \\
\hline$\varnothing$ \\
\hline$\varnothing$ \\
\hline
\end{tabular}

When two such structures come together they will be merged. The merge operation $\oplus$ takes two structures and results in a new one thereby using the referent systems to substitute tho names of referents if necessary and then taking the union of the sets of clauses. E.g. the result of the merge $/ \mathrm{g} / \oplus / 2 /$ is

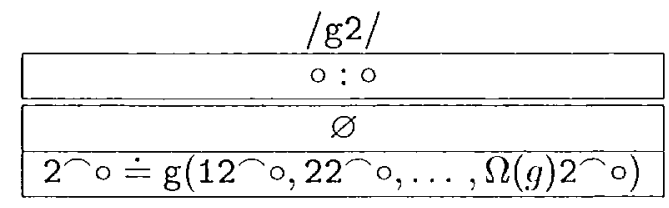

The meaning of $\circ: 2 \frown 0$ is as follows. If somo structure $A$ is merged with one bearing that refcrent system, then all occurrences of the variable $\circ$ in $A$ are replaced by $2-0$. As the resulting referent system we get $0: 0$. This is exactly what is done in the merge shown above. We shall call a structure with referent system $\circ$ : o plain. Merge is only defined if at least on structure is plain.

\subsection{Semantics for $\mathcal{I C M L}$}

T.o sec how the semantics works we shall reproduce an earlier example and take the $\mathcal{I C M} \mathcal{L}^{\Omega}$ string $\mathrm{g} 2 \times 1 \mathrm{f} \times 12$. Motivated by the definition of the ideal case marking language we shall agree to the conventions that

1. Case markers may only be suffixes

2. Case markers may only be attached to functors or case marked functors

By these conventions the string under considcration must be parsed as $(g 2)(x 1)(f)((x 1) 2)$. They force us to combine the functors with their case stacks first and afterwards combine the units. We shall understand that this is a syntactic restriction and not due to any semantics.

The composition of $\mathrm{g}$ and 2 was already shown above and is repeated on the left hand side, using that $\Omega(g)=1$. The result of composing $\mathrm{x}$ and 1 is shown to the right. 

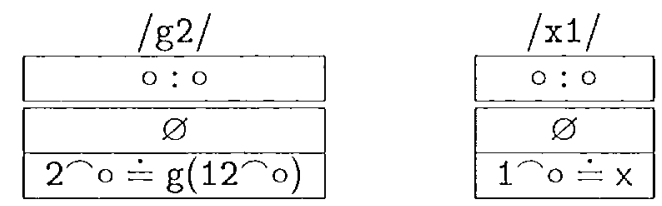

Merging these two structures we get

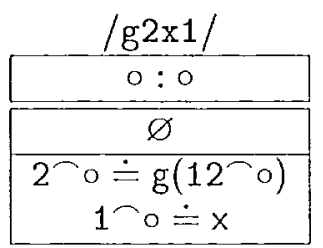

Together with $f$ this gives

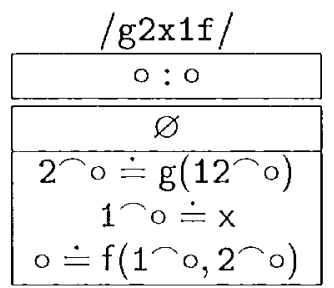

By composing the structures for $\mathrm{x}$ and 1 we get the structure $/ \mathrm{x} 1 /$ shown above. We merge this one with that for 2 and get

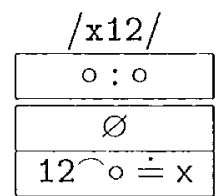

The merge of the two structures above finally gives

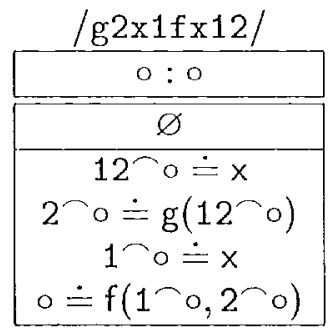

We shall verify that the value of $\circ$ is actually the same as the value of $f(x, g(x))$. Notice first that in the body of the DRS we find that $12{ }^{\circ} \mathrm{O}$ and 1 o have the same value as $x$. We may therefore reduce the body of this structure to

$$
\begin{gathered}
2 \frown \mathrm{o} \doteq \mathrm{g}(\mathrm{x}) \\
\mathrm{o} \doteq \mathrm{f}\left(\mathrm{x}, 2{ }^{\frown} \mathrm{o}\right)
\end{gathered}
$$

Finally we may replace 2 o by $g(x)$ in the second line. We get then

$$
0 \doteq \mathrm{f}(\mathrm{x}, \mathrm{g}(\mathrm{x}))
$$

which is the intended result.

After the semantics of the mits has been computed the order of merge is umimportant. If we choose to merge these semanties in an order different from the one above, we gret the same result.

\subsection{An example from Warlpiri}

To show how this proposal may work for natural languages we give an example from Warlpirit in which case stacking occurs. We have to deal with the four case markers crgative (ERG), past tense (PST), absolutive (ABS), and locative (LOC).

Japanangka-rlu luwa-rnu marlu Japanangka-ERG shoot-PS'T kangaroo-Aiss pirli-ngkia-rlu

rock-LOC-ERG

Japanangka shot the kangaroo (while) on the rock

We oxtend the proposal by taking into account that cases may not only function as argument markers but have a semantics, too. This actually does not make much of a diflerence for this calculus. We propose the following semanties for the locative and the past tense case marker
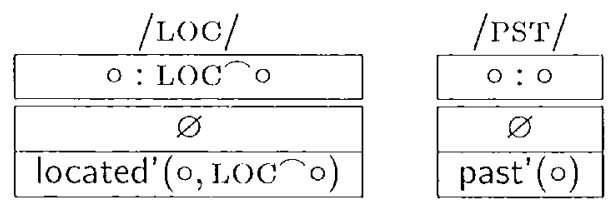

So, when the locative is attached, it says that the thing to which it attaches is located somewhere. Hore, o represents the thing that is located, while doC $\mathrm{O}$ is the location. The past tense scmantics simply says that the thing which it attaches to happened in the past.

Wo construe the meaning of the ergative as being the actor and the meaning of the absolutive as being the theme ${ }^{5}$.

\footnotetext{
${ }^{4}$ This example is taken from (Nordlinger, 1997), p.171

${ }^{5}$ In fact, crgative and absolutive should mark for grammatical functions, but since linking of grammatical functions and actants is cuite a complicated matter (see (Kracht, 1999)) we make this simplification.
} 


\begin{tabular}{|c|}
\multicolumn{1}{c|}{$/$ ERG $/$} \\
\hline $0:$ ERG \\
\hline$\varnothing$ \\
\hline actor $^{\prime}(0) \doteq \mathrm{ERG}^{\frown 0}$ \\
\hline theme $(0) \doteq \mathrm{ABS}^{\circ} \mathrm{O}$ \\
\hline
\end{tabular}

Again, we shall agree in using the conventions stated in subsection 3.2. First we have to attach the case markers to compose the resulting structures afterwards. The semantics of the proper noun Japanangka is taken to be a plain structure with body o $\doteq$ japanangka'. The composition of this structure and the ergative semantics yiclds

/Japanangka-ERG/
\begin{tabular}{|c|}
\hline $0: 0$ \\
\hline$\varnothing$ \\
\hline $\begin{array}{c}\text { ERG }^{\sim} 0 \doteq \text { japanangka }^{\prime} \\
\text { actor }^{\prime}(0) \doteq \text { ERG }^{\prime} \mathrm{O}\end{array}$ \\
\hline
\end{tabular}

We assume that the nominals / pirlif and /marlu/ have the following lexical entries:

\begin{tabular}{|c|}
\multicolumn{1}{l}{$\mid$ pirli| } \\
\hline $0: 0$ \\
\hline \hline$\{0\}$ \\
\hline $\operatorname{rock}^{\prime}(0)$ \\
\hline
\end{tabular}

\begin{tabular}{|c|}
\multicolumn{1}{c|}{$/$ marlu/ } \\
\hline $0: 0$ \\
\hline ko\} \\
\hline kangaroo' $(0)$ \\
\hline
\end{tabular}

Thus the semantic composition of pirli, LOC, and ERG gives:

\begin{tabular}{|c|}
\hline /pirli-LOC-ERG/ \\
\hline $0: 0$ \\
\hline$\{\mathrm{LOC}$ ERG O $\}$ \\
\hline $\begin{array}{l}\operatorname{rock}^{\prime}\left(\mathrm{IOC}_{\mathrm{ORG}} \mathrm{O}\right) \\
\text { actor }(\mathrm{O}) \doteq \mathrm{ERG} \mathrm{O}^{\circ}\end{array}$ \\
\hline
\end{tabular}

Similarly we can compose marlu with the absolutive case:

\begin{tabular}{|c|}
\hline /marlu-ABS/ \\
\hline $0: 0$ \\
\hline$\{\mathrm{ABS} \frown \mathrm{O}\}$ \\
\hline $\begin{array}{l}\text { kangaroo' }\left(\mathrm{ABS}^{\circ} \mathrm{O}\right) \\
\text { theme }(\mathrm{O}) \stackrel{\mathrm{ABS}}{\doteq} \mathrm{O}\end{array}$ \\
\hline
\end{tabular}

The semantics of the verb is shown on the left hand side and its composition with /PST/ on the right hand side.

\begin{tabular}{|c|c|}
\multicolumn{1}{c|}{$/$ /luwa/ } & /uwa-PST/ \\
\hline $0: 0$ \\
\hline$\varnothing$ \\
\hline shoot $^{\prime}(0)$ \\
\hline
\end{tabular}$\quad$\begin{tabular}{c}
$\varnothing$ \\
\hline $\begin{array}{c}\text { shoot' }^{\prime}(0) \\
\text { past' }^{\prime}(0)\end{array}$ \\
\hline
\end{tabular}

Finally by merging the structures / JapanangkaERG/, /luwa-PST/, /marlu-ABS/, and /pirliLOC-ERG/ in any order, we get the following result.

\begin{tabular}{|c|}
\hline $0: 0$ \\
\hline$\left\{\mathrm{ABS}^{\prime} \circ, \mathrm{LOC} \mathrm{ERG} \circ\right\}$ \\
\hline 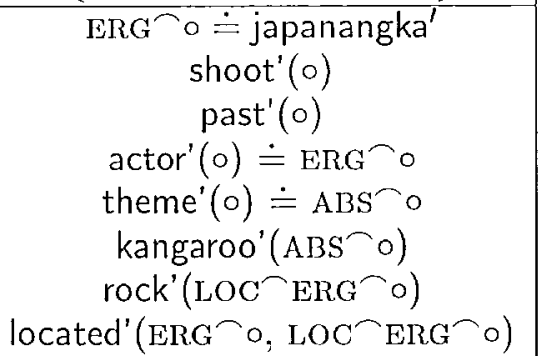 \\
\hline
\end{tabular}

It says that there was an event of shooting in the past, whose actor is Japanangka and whose theme is something that is a kangaroo, and that there is a rock, such that Japanangka is located on it. Note that the only syntactic restriction were the conventions stated in subsection 3.2 and that we did not make any further assumptions on syntactic structure or word order.

\section{References}

Annius Groenink. 1997. Surface without structure. Ph.D. thesis, Centrum voor Wiskunde en Informatica, Amsterdam.

Marcus Kracht. 1999. Agreement morphology, argument structure and syntax. Course material ESSLLI '99.

Robert Malouf. 1999. A head-driven account of long-distance case assignment. Paper presented on HPSG' 99 .

Jens Michaelis and Marcus Kracht. 1997. Semilinearity as a syntactic invariant. In Christian Retoré, editor, Proceedings of the 1st International Conference on Logical Aspects of Computational Linguistics (LACL-96), volume 1328 of LNCS, pages 329-345, Berlin, September 23-25. Springer.

Rachel Nordlinger. 1997. Constructive Case. Dependent-Marking Nonconfigurality in Australia. Ph.D. thesis, Department of Linguistics, Stanford University, Stanford.

Kces F. M. Vermeulen. 1995. Morging without mystery or: Variables in dynamic semantics. Journal of Philosophical Logic, 24:405-450.

David J. Weir. 1988. Characterizing Mildly Context-Sensitive Grammar Formalisms. Ph.D. thesis, University of Pennsylvania, PA. 